\title{
Current-induced magnetization reversal in nanopillars with perpendicular anisotropy
}

\author{
S. MANGIN ${ }^{1,2}$, D. RAVELOSONA ${ }^{1,3}$, J. A. KATINE ${ }^{1}$, M. J. CAREY ${ }^{1}$, B. D. TERRIS ${ }^{1}$ AND \\ ERIC E. FULLERTON ${ }^{1 *}$ \\ ${ }^{1}$ Hitachi Global Storage Technologies, San Jose Research Center, 650 Harry Road, San Jose, California 95120, USA \\ ${ }^{2}$ Laboratoire de Physique de Matériaux, UMR CNRS 7556, U.H.P.-Nancy I, B.P. 239 F-54506 Vandoeuvre cedex, France \\ ${ }^{3}$ Institut d'Electronique Fondamentale, UMR CNRS 8622, Université Paris Sud, 91405 Orsay Cedex, France \\ *e-mail: eric.fullerton@hgst.com
}

Published online: 19 February 2006; doi:10.1038/nmat1595

Devices that show a magnetic anisotropy normal to the film surface hold great promise towards faster and smaller magnetic bits in data-storage applications. We describe an experimental demonstration of current-induced magnetic reversal of nanopillars with perpendicular anisotropy and high coercive fields. The best results are observed for Co/Ni multilayers, which have higher giant magnetoresistance values and spin-torque efficiencies than Co/Pt multilayers. The reference layers were designed to have significantly higher anisotropy allowing a complete current-field phase diagram of the free-layer reversal to be explored. The results are compared to micromagnetic modelling of the free layer that, depending on the bias current and applied field, details regions of irreversible magnetic switching, coherent and incoherent spin waves, or static non-uniform magnetization states. This ability to manipulate high-anisotropy magnetic elements could prove useful for a range of spintronic applications.
$(0)$ ince the theoretical prediction ${ }^{1,2}$ of spin-wave excitations and magnetization reversal of nano-elements driven by polarized spin currents, and its subsequent observation in point contact $^{3}$ and geometries of patterned devices ${ }^{4-6}$, this subject has generated considerable experimental (see, for instance, ref. 7) and theoretical interest (see, for instance, ref. 8). The basic phenomena occur for current flowing through two magnetic elements separated by a thin non-magnetic spacer layer. The current becomes spin polarized by transmission through or on reflection from the first magnetic layer (the reference layer) and mostly maintains this polarization as it passes through the non-magnetic spacer and enters and interacts with the second ferromagnetic layer (the free layer). This interaction leads to a change of resistance depending on the relative orientation of the magnetic layers known as giant magnetoresistance (GMR), which is the basis of many current magnetic devices ${ }^{9}$. Commensurate with the GMR, there is a transfer of angular momentum from the polarized current to the free-layer magnetization that can be described as an effective torque. This spin torque can oppose the intrinsic damping of the magnetic layer, exciting spin waves and, for sufficient current strengths, reversing the direction of the magnetization ${ }^{1-8}$.

Although such studies give a deeper understanding of the interaction between electrons and magnetization that give rise to effects such as $\mathrm{GMR}^{10-12}$, they also provide the impetus for many emerging spintronic technologies. One attractive application is direct switching of magnetic bits in a magnetic memory cell by local application of current, rather than by the magnetic fields generated by external wires ${ }^{13}$. The practical use of such technologies requires the efficiency and speed of the spin-torque switching of the magnetic element to be improved such that high-anisotropy, thermally stable bits can be written reliably. Although several approaches have been put forward to enhance the efficiency for in-plane magnetized elements used at present ${ }^{14-16}$, one particularly elegant approach is to design elements with the magnetic anisotropy normal to the film surface. For devices with the reference layer having perpendicular magnetization and the free layer having in-plane anisotropy, there are predictions for an 
increase in the speed of switching ${ }^{17,18}$. In this article we demonstrate magnetic reversal driven by spin currents in structures with both the reference and free layer possessing perpendicular magnetic anisotropy. These results are enabled by a materials design of $\mathrm{Co} / \mathrm{Ni}$ multilayers that combine both high spin polarization and perpendicular magnetic anisotropy. We also map out a currentfield phase diagram for reversal of the free layer and compare the results to micromagnetic modelling.

The anticipated efficiency enhancement for an allperpendicular structure is estimated theoretically by comparing the critical currents for spin-torque reversal and thermal stability of in-plane versus out-of-plane anisotropy devices. The critical currents for reversal can be estimated from a stability analysis of the Landau-Lifshitz-Gilbert (LLG) equations of motion, including the Slonczewski form of the spin-transfer torque ${ }^{2,6,10}$. For inplane magnetized devices, the critical currents for switching from the parallel-to-antiparallel (P-AP) and the antiparallel-to-parallel (AP-P) configurations are given, respectively, by

$$
\begin{gathered}
I_{\mathrm{C}}^{\mathrm{P}-\mathrm{AP}} \approx \frac{A \alpha M_{\mathrm{S}} V}{g(0) p}\left(H+H_{\mathrm{dip}}+H_{K \|}+2 \pi M_{\mathrm{S}}\right) \quad \text { and } \\
I_{\mathrm{C}}^{\mathrm{AP}-\mathrm{P}} \approx \frac{A \alpha M_{\mathrm{S}} V}{g(\pi) p}\left(H+H_{\mathrm{dip}}-H_{K \|}-2 \pi M_{\mathrm{S}}\right)
\end{gathered}
$$

where $M_{\mathrm{s}}, V$ and $\alpha$ are the saturation magnetization, volume and Gilbert damping constant for the free layer, respectively, and $p$ is the spin polarization of the current. The factor $g$ depends on the relative angle of the reference-layer and free-layer magnetizations, where the specific angular dependence is dependent on $p$ (ref. 2). The factor $A$ is dependent on the specifics of the transport model, but is of the order of $3 \times 10^{11} \mathrm{~mA} \mathrm{Oe}{ }^{-1}$ e.m.u. ${ }^{-1} . H, H_{\text {dip }}$ and $H_{K \|}$ are the in-plane applied field, the dipole field from the reference layer acting on the free layer and the uniaxial in-plane anisotropy field, respectively. The factor $2 \pi M_{\mathrm{S}}$ arises from the demagnetizing field of the thin-film geometry. For the out-of-plane case, the critical currents are given by

$$
\begin{gathered}
I_{\mathrm{C}}^{\mathrm{P}-\mathrm{AP}} \approx \frac{A \alpha M_{\mathrm{S}} V}{g(0) p}\left(H_{K \perp}-4 \pi M_{\mathrm{S}}-H-H_{\mathrm{dip}}\right) \quad \text { and } \\
I_{\mathrm{C}}^{\mathrm{AP}-\mathrm{P}} \approx \frac{A \alpha M_{\mathrm{S}} V}{g(\pi) p}\left(-H_{K \perp}+4 \pi M_{\mathrm{S}}-H-H_{\mathrm{dip}}\right),
\end{gathered}
$$

where $H_{K \perp}$ is the perpendicular uniaxial anisotropy field of the free layer, and $H$ and $H_{\text {dip }}$ are now fields perpendicular to the film. For equation (2) we assume that the reference layer has it magnetization in the negative direction (Fig. 1) and gives the negative pre-factor to $H$. In this geometry the demagnetizing field is collinear to the anisotropy field giving an effective perpendicular anisotropy field $H_{K}=H_{K \perp}-4 \pi M_{\mathrm{S}}$ where $H_{K}$ must be greater than zero for perpendicular remanence.

The energy barrier of the bit against thermal fluctuation of the magnetization is $M_{\mathrm{S}} V H_{K \|} / 2$ for the in-plane device, whereas it is $M_{\mathrm{S}} V\left(H_{K \perp}-4 \pi M_{\mathrm{S}}\right) / 2$ for the perpendicular device. Thus, one potential advantage to the perpendicular geometry is that the critical currents for switching the magnetization (for small $H$ and $H_{\text {dip }}$ ) are directly proportional to anisotropy, and, hence, the stability of the bit. For the in-plane devices, the current must overcome the additional additive factor $2 \pi M_{\mathrm{S}}$ that does not contribute to the stability of the bit against thermal fluctuations, but suppresses spin-torque induced switching. However, to take advantage of the perpendicular geometry, one needs a materials

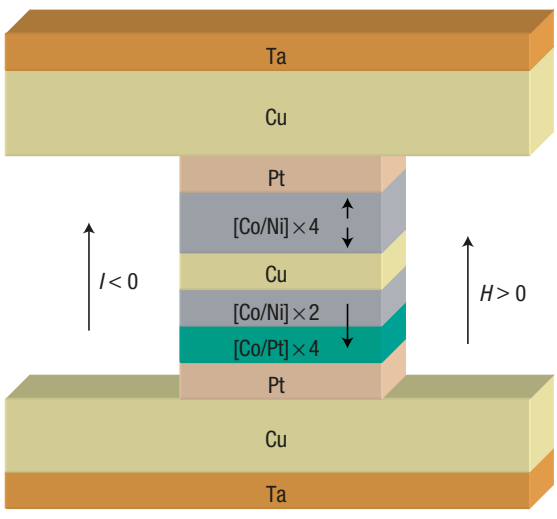

Figure 1 Schematic representation of patterned Co/Ni samples. The reference layer is a composite $[\mathrm{Co} / \mathrm{Pt}] \times 4 /[\mathrm{Co} / \mathrm{Ni}] \times 2$ multilayer and the free layer is a $[\mathrm{Co} / \mathrm{Ni}] \times 4$ multilayer. The magnetization direction of the reference layer, positive field direction and the direction of electron flow for negative current are shown.

system with perpendicular anisotropy while maintaining the other parameters such as $p$ and $\alpha$.

So far, few or no experimental spin-transfer results have been published on perpendicular anisotropy elements. The closest work is the study of spin-torque switching for planar anisotropy samples in large perpendicular fields ${ }^{19-22}$. In our samples, both magnetic layers have perpendicular anisotropy sufficient to overcome the shape anisotropy and maintain the magnetization perpendicular to the film in the absence of an applied field. For this we used $\mathrm{Co} / \mathrm{Ni}$ and/or $\mathrm{Co} / \mathrm{Pt}$ multilayers. For many perpendicularly magnetized devices, $\mathrm{Co} / \mathrm{Pt}$ (or $\mathrm{Co} / \mathrm{Pd}$ ) multilayers are used where the $\mathrm{Co}-\mathrm{Pt}$ interfacial anisotropy provides the perpendicular anisotropy. However, for spin-transport devices, the incorporation of weakly or non-magnetic Pt layers within the magnetic layers leads to high spin-orbit scattering and reduced spin-torque efficiencies. As such, we focus on devices with $\mathrm{Co} / \mathrm{Ni}$ multilayers as the magnetic layers. These multilayers contain only magnetic atoms and have perpendicular anisotropy for thin $\mathrm{Co}$ and $\mathrm{Ni}$ layers ${ }^{23}$. The $\mathrm{Co} / \mathrm{Ni}$ sample structure is shown schematically in Fig. 1 . The reference layer is a composite $\mathrm{Co} / \mathrm{Pt}$ and $\mathrm{Co} / \mathrm{Ni}$ multilayer to enhance the anisotropy and the free layer is a Co/Ni multilayer. For comparison, we also fabricated devices based solely on $\mathrm{Co} / \mathrm{Pt}$ multilayers.

We designed the reference layer to have higher anisotropy than the free layer allowing us to access both parallel and antiparallel alignments of the magnetic layers with applied field. Magnetic measurements performed before the patterning process for fields applied perpendicular to the film revealed full perpendicular magnetic remanence and abrupt reversal of each layer with no coupling between the layers. The reference-layer and free-layer coercive fields were 2.75 and $0.75 \mathrm{kOe}$, respectively, for the $\mathrm{Co} / \mathrm{Ni}$ sample (2.9 and $1.8 \mathrm{kOe}$, respectively, for the $\mathrm{Co} / \mathrm{Pt}$ sample). Samples without the $\mathrm{Cu}$ leads were grown to measure the GMR of the full film in a current-in-plane geometry. The $\mathrm{Co} / \mathrm{Ni}$ and $\mathrm{Co} / \mathrm{Pt}$ structures had GMR ratios of $2.5 \%$ and $0.5 \%$, respectively, demonstrating the effectiveness of the $\mathrm{Co} / \mathrm{Ni}$ layers in enhancing the GMR signal.

In the remainder of the discussion we focus primarily on $\mathrm{Co} / \mathrm{Ni}$ devices of hexagonal shapes that are nominally $50 \times 100 \mathrm{~nm}^{2}$. The differential resistance $\mathrm{d} V / \mathrm{d} I$ versus perpendicular magnetic field $H$ measured at room temperature is plotted in Fig. 2a. The layers switch in discrete jumps between the antiparallel (high- 

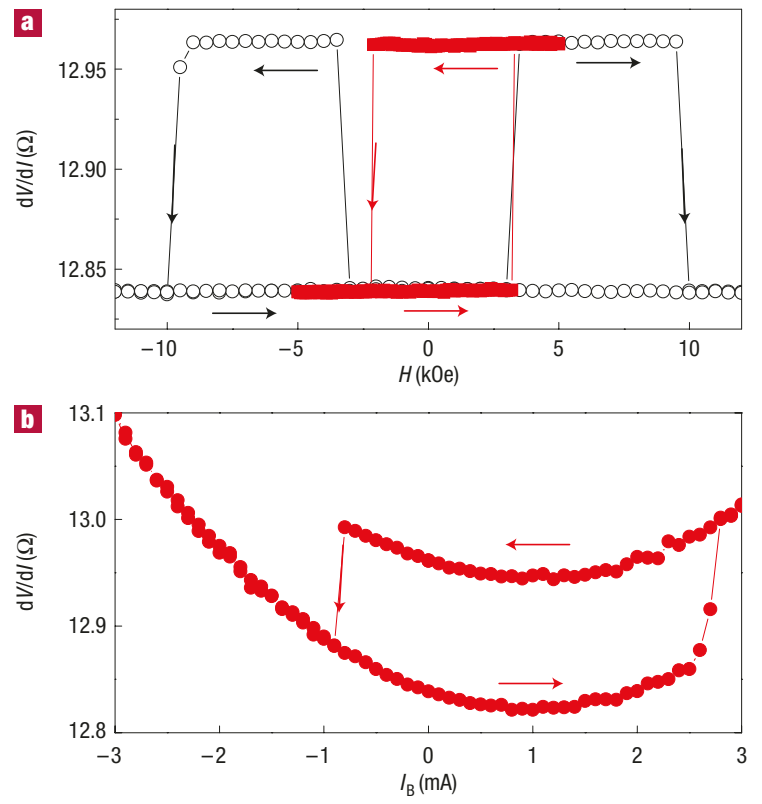

Figure 2 Transport measurements of the $50 \times 100 \mathrm{~nm}^{2}$ Co/Ni sample. a, $\mathrm{d} V / \mathrm{d} /$ versus $H$ for $H$ perpendicular to the film plane. The open symbols correspond to the major loop showing discrete transitions between the parallel and antiparallel states. The filled symbols are a minor loop where only the free layer reverses with the reference-layer magnetization pointing down. $\mathbf{b}, \mathrm{dV} / \mathrm{d} /$ versus $I_{\mathrm{B}}$ for $H=0$ showing discrete transitions between the antiparallel and parallel states.

resistance) and parallel (low-resistance) alignment with a current perpendicular to the plane GMR ratio of $1.0 \%$. The coercive field of both layers have increased significantly over the continuous film values. The reference layer now has a coercive field of $10 \mathrm{kOe}$ and the free layer $2.65 \mathrm{kOe}$. This is in accordance with observations on patterned perpendicular media, where the coercive field increases as one moves from reversal by means of domain nucleation and growth in a full film towards coherent rotation in patterned structures $^{24}$. In Fig. 2a, the minor hysteresis loop shows the reversal of only the free layer, which is offset by 650 Oe from the origin owing to the average dipolar field $\left(H_{\text {dip }}\right)$ from the lower layer that favours parallel alignment.

The $\mathrm{d} V / \mathrm{d} I$ versus the d.c. current bias $\left(I_{\mathrm{B}}\right)$ curve is shown in Fig. $2 \mathrm{~b}$ for $H=0$. Starting from the parallel alignment, the reference layer switches into the antiparallel configuration for $I_{\mathrm{C}}^{\mathrm{P}-\mathrm{AP}}=2.7 \mathrm{~mA}$ $\left(7.5 \times 10^{7} \mathrm{~A} \mathrm{~cm}^{-2}\right)$ and switches back for $I_{\mathrm{C}}^{\mathrm{AP}-\mathrm{P}}=-0.85 \mathrm{~mA}$ $\left(-2.6 \times 10^{7} \mathrm{~A} \mathrm{~cm}^{-2}\right)$. Measurements for eight devices (with areas varying by a factor of four) yielded similar critical current densities; $I_{\mathrm{C}}^{\mathrm{P}-\mathrm{AP}}$ ranging from 7.5 to $11.0 \times 10^{7} \mathrm{~A} \mathrm{~cm}^{-2}$ and $I_{\mathrm{C}}^{\mathrm{AP}-\mathrm{P}}$ ranging from -2.6 to $-4.5 \times 10^{7} \mathrm{~A} \mathrm{~cm}^{-2}$. The resistance and coercivity of our $\mathrm{Co} / \mathrm{Pt}$ devices scaled with reduced lateral dimensions in a manner very similar to our $\mathrm{Co} / \mathrm{Ni}$ samples. However, the $\mathrm{Co} / \mathrm{Pt} \mathrm{GMR}$ ratio was only $0.4 \%$ and the current density needed to drive free-layer reversal was three to four times higher than in the $\mathrm{Co} / \mathrm{Ni}$ samples.

Starting from either the antiparallel or parallel alignment of the magnetic layers, a series of $\mathrm{d} V / \mathrm{d} I$ versus $I_{\mathrm{B}}$ and $\mathrm{d} V / \mathrm{d} I$ versus $H$ scans were measured to construct the $I_{\mathrm{B}}-H$ phase diagram shown in Fig. 3. For this phase diagram the lower (reference) layer has its magnetization pointing down and for the current-field range shown, only the upper (free) layer reverses. The high coercive field of the reference layer allows the reversal of the free layer to be

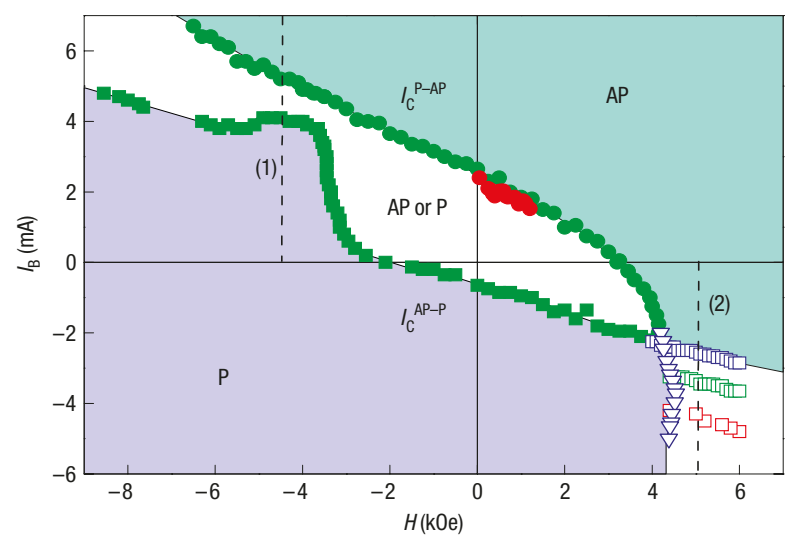

Figure 3 Experimentally determined $I_{\mathrm{B}}-H$ phase diagram for the $50 \times 100 \mathrm{~nm}^{2} \mathrm{Co} / \mathrm{Ni}$ sample showing the parallel or antiparallel states where each symbol corresponds to a distinct change in the resistance. The filled symbols are irreversible changes where the circles are $I_{C}^{\mathrm{P}-\mathrm{AP}}$ and squares are $I_{\mathrm{C}}^{\mathrm{AP}-\mathrm{P}}$. The filled red circles indicate a double transition at $I_{C}^{\mathrm{P}-\mathrm{AP}}$. The open symbols refer to reversible changes in the resistance. Lines labelled (1) and (2) correspond to the $I_{B}$ scans in Fig. 4a and b, respectively. See text for further discussion.

studied with large applied fields either parallel or antiparallel to the reference layer in a regime not accessible in previous studies ${ }^{7,19-22}$. In Fig. 3, each symbol corresponds to a discrete change in the resistance while changing $H$ or $I_{\mathrm{B}}$. We discuss the results in Fig. 3 in detail below; however, the general features of the phase diagram are seen in a second $50 \times 100 \mathrm{~nm}^{2}$ device and $100 \times 200 \mathrm{~nm}^{2}$ hexagonal and $100 \mathrm{~nm}$ diameter circular devices. For $H<4.2 \mathrm{kOe}$, we observe hysteretic reversal of magnetization between the antiparallel and parallel states where the filled squares correspond to the critical bias current for AP-P transitions $I_{\mathrm{C}}^{\mathrm{AP}-\mathrm{P}}$ and the filled circles correspond to $\mathrm{P}-\mathrm{AP}$ transitions $I_{\mathrm{C}}^{\mathrm{P}-\mathrm{AP}}$. For $H>4.2 \mathrm{kOe}$, we observe reversible changes in the resistance given by open symbols.

Although equation (2) is only strictly valid at low temperatures, portions of our room-temperature phase diagram agree with its predictions. For $H<2.0 \mathrm{kOe}, I_{\mathrm{C}}^{\mathrm{P}-\mathrm{AP}}$ follows the linear $H$ dependence (slope $=-6.4 \times 10^{-4} \mathrm{~mA} \mathrm{Oe}{ }^{-1}$ ) and intercepts $I_{\mathrm{B}}=0$ near $H=H_{\text {dip }}+H_{K}$, which is roughly the positive coercive field. Similarly, $I_{\mathrm{C}}^{\mathrm{AP}-\mathrm{P}}$ follows a linear behaviour for $H>-3.0 \mathrm{kOe}$ and intercepting the $I_{\mathrm{B}}=0$ axis at $H=-H_{K}+H_{\text {dip }}$ near the negative coercive field (slope $\left.=-4.0 \times 10^{-4} \mathrm{~mA} \mathrm{Oe}^{-1}\right)$. The slopes correspond to the pre-factor in equation (2). From the slope we can estimate $\alpha / p=0.20 g(\pi)$ and $\alpha / p=0.32 g(0)$. Given that $g(\theta)$ is expected to range between 0.1 and 0.5 , the values of $\alpha / p$ are comparable with values determined for in-plane devices. The corresponding value for the $\mathrm{Co} / \mathrm{Pt}$ devices was $\alpha / p=0.80 g(\pi)$, indicating reduced polarization and/or increased damping as compared with the $\mathrm{Co} / \mathrm{Ni}$ samples consistent with the reduced GMR values.

In other parts of the phase diagram, the data deviate from the linear behaviour of equation (2). For $H<-3.0 \mathrm{kOe}$, there is a sharp upturn in $I_{\mathrm{C}}^{\mathrm{AP}-\mathrm{P}}$, which is then non-monotonic with $H$ reaching a peak at $H=-4.5 \mathrm{kOe}$. Similarly, there is a sharp downturn in $I_{\mathrm{C}}^{\mathrm{P}-\mathrm{AP}}$ for $H>3.0 \mathrm{kOe}$. This behaviour can be explained, in part, by finite temperature effects ${ }^{25}$. However, thermal activation alone does not explain the non-monotonic response of $I_{\mathrm{C}}^{\mathrm{AP}-\mathrm{P}}$ for negative applied fields. Commensurate with the nonmonotonic $H$ dependence, we observe a change in the resistance before $I_{\mathrm{C}}^{\mathrm{AP}-\mathrm{P}}$ for $-5.0<H<-4.0 \mathrm{kOe}$. This is shown in Fig. 4a, 

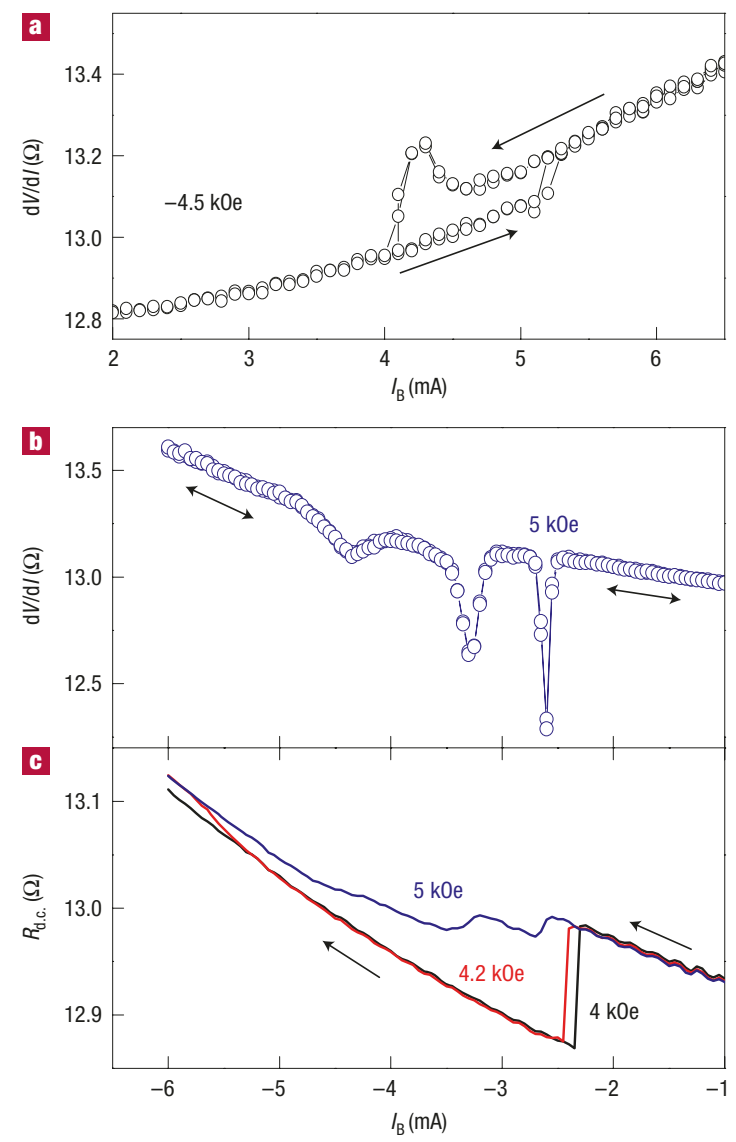

Figure 4 Transport measurements of the $50 \times \mathbf{1 0 0} \mathrm{nm}^{2}$ Co $/$ Ni sample. a, $\mathrm{d} V / \mathrm{d} /$ versus $I_{\mathrm{B}}$ for $H=-4.5 \mathrm{kOe}$ corresponding to the line (1) in Fig. 3 . The arrows indicate current sweep directions. $\mathbf{b}, \mathrm{d} V / \mathrm{d} /$ versus $I_{\mathrm{B}}$ for $H=5.0 \mathrm{kOe}$ corresponding to line (2) in Fig. 3. The scan is reversible with current directions.

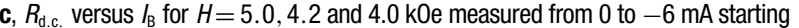
from the antiparallel configuration.

which corresponds to the $I_{\mathrm{B}}$ scan shown as line (1) in Fig. 3. For increasing current from the parallel state there is a discrete jump up in $\mathrm{d} V / \mathrm{d} I$ for $I_{\mathrm{C}}^{\mathrm{P}-\mathrm{AP}}$. However, there is a distinct upturn and peak in $\mathrm{d} V / \mathrm{d} I$ before the $I_{\mathrm{C}}^{\mathrm{AP}-\mathrm{P}}$ transition as the current is subsequently reduced. This upturn in $\mathrm{d} V / \mathrm{d} I$ is reversible and demonstrates a current-induced reduction in the d.c. resistance $\left(R_{\mathrm{d} . c .}\right)$ before the irreversible transition occurs. This behaviour is reproduced, in part, in micromagnetic simulations described below. Another interesting regime is $0<H<1.0 \mathrm{kOe}$ where $I_{\mathrm{C}}^{\mathrm{P}-\mathrm{AP}}$ is characterized by two jumps where an intermediate resistance state is observed. The second transitions are shown as the red circles in Fig. 3 and correspond to a domain state and will be discussed elsewhere.

For $H>4.2 \mathrm{kOe}$ we observe magnetic transitions that are reversible in either $H$ or $I_{\mathrm{B}}$ scans and give sharp dips in $\mathrm{d} V / \mathrm{d} I$. Such dips or peaks are often related to magnetic excitations of the free layer ${ }^{6}$. From examining equation (2) it is clear that for $H>H_{\text {dip }}+H_{K}(g(\pi) / g(0)+1) /(g(\pi) / g(0)-1)$ one reaches the field range where $I_{\mathrm{C}}^{\mathrm{P}-\mathrm{AP}}<I_{\mathrm{C}}^{\mathrm{AP}-\mathrm{P}}$. In the region $I_{\mathrm{C}}^{\mathrm{P}-\mathrm{AP}}<I_{\mathrm{B}}<I_{\mathrm{C}}^{\mathrm{AP}-\mathrm{P}}$, neither uniform magnetized state is stable and spin-wave modes are expected. In this region, we observe a series of reversible transitions that are manifested as three sharp dips in $\mathrm{d} V / \mathrm{d} I$ versus $I_{\mathrm{B}}$, where the position of the peaks is represented by the differently coloured open squares in Fig. 3. In Fig. $4 \mathrm{~b}$ and $\mathrm{c}$, we show both $\mathrm{d} V / \mathrm{d} I$ and $R_{\text {d.c. }}$ as a function of $I_{\mathrm{B}}$ for $H=5.0 \mathrm{kOe}$. The field dependence of the modes is consistent with equation (2) . Examining Fig. 4c, we see that each transition corresponds to an $R_{\text {d.c. }}$ change of about $0.3 \%$. The origin of the transitions is not understood completely. They could arise from lateral variation in the sample with each part of the sample having distinct critical currents. However, given the common slope with $H$ for all of the modes, this explanation is unlikely. More likely is that each transition corresponds to a uniform spin-wave mode where the angle of procession increases in discrete steps with increasing current densities.

Also shown in Fig. $4 \mathrm{c}$ are the $R_{\text {d.c. }}$ versus $I_{\mathrm{B}}$ curves for 4.2 and $4.0 \mathrm{kOe}$ fields. The $H=4.0 \mathrm{kOe}$ curve shows a single, discrete transition from the antiparallel to parallel configuration. Comparing the $H=4.0$ and $5.0 \mathrm{kOe}$ scans, it is clear for the $5.0 \mathrm{kOe}$ scan that the $R_{\text {d.c. }}$ of the parallel configuration is not reached even at the highest negative $I_{\mathrm{B}}$. In addition to the reversible modes discussed above, we also observe a nearly vertical reversible transition line for $H=4.2 \mathrm{kOe}$ and large negative currents ( $\nabla$ in Fig. 3). The manifestation of this transition can be seen in Fig. 4c for the $H=4.2 \mathrm{kOe}$ curve. With decreasing $I_{\mathrm{B}}$, the discrete resistance jump at $I_{\mathrm{C}}^{\mathrm{AP}-\mathrm{P}}$ is observed at $I_{\mathrm{B}}=-2.4 \mathrm{~mA}$. However, at $I_{\mathrm{B}}=$ $-5.5 \mathrm{~mA}$ the resistance increases to values similar to those observed for higher fields and away from the parallel configuration.

To gain a fuller understanding of our results, we performed an LLG micromagnetic calculation of our system including the Slonczewski spin-torque term ${ }^{26}$. In Fig. 5, we plot the resulting phase diagram where the symbols correspond to either a reversal of the free-layer magnetization (filled symbols) or deviation from a uniform magnetization (open symbols). Within these calculations we observe trends similar to our experimental results. $I_{\mathrm{C}}^{\mathrm{AP}-\mathrm{P}}$ and $I_{\mathrm{C}}^{\mathrm{P}-\mathrm{AP}}$ follow, for the most part, the expected linear $H$ dependence of equation (2) shown as the red lines. For the $I_{\mathrm{C}}^{\mathrm{AP}-\mathrm{P}}<I_{\mathrm{C}}^{\mathrm{P}-\mathrm{AP}}$ $(H<5.0 \mathrm{kOe})$ we observe hysteretic switching. For large positive fields $(H>6.0 \mathrm{kOe})$ where equation (2) predicts $I_{\mathrm{C}}^{\mathrm{AP}-\mathrm{P}}>I_{\mathrm{C}}^{\mathrm{P}-\mathrm{AP}}$, we see the onset of spin waves that mostly correspond to uniform precessional modes (labelled SW in Fig. 5). The transitions from the uniform magnetization to spin-wave modes are shown by the open circles and squares and occur at the linear $I_{\mathrm{C}}^{\mathrm{AP}-\mathrm{P}}$ and $I_{\mathrm{C}}^{\mathrm{P}-\mathrm{AP}}$ phase lines. In between $I_{\mathrm{C}}^{\mathrm{AP}-\mathrm{P}}$ and $I_{\mathrm{C}}^{\mathrm{P}-\mathrm{AP}}$ the angle of precession increases until a critical current where the average magnetization reverses from antiparallel to parallel alignments (or vice versa) by means of the formation of chaotic spin waves similar to those described in ref. 27, before again forming a uniform processional mode that decreases its angle as the current approaches the second phase boundary. The current where the average free-layer magnetization switches between antiparallel to parallel alignments is shown by the dashed line.

In addition to hysteretic switching and spin-wave modes in the simulations, we find the occurrence of static non-uniform canting of the spins in the free layer. In this mode, the spins along the edge of the free layer fan out away from the surface-normal direction. This mode is reversible with $I_{\mathrm{B}}$ or $H$ and occurs in the regions outlined by the open triangles in Fig. 5. For positive field, this occurs for large negative $I_{\mathrm{B}}$. The open triangles $(\nabla)$ indicate the onset of this canted mode and has an expected change of resistance of about $10 \%$ of the full GMR ratio. For negative fields, we only observe the canted mode in a narrow $I_{\mathrm{B}}-H$ window $(\triangle)$ that occurs before reversal of the free layer at $I_{\mathrm{C}}^{\mathrm{AP}-\mathrm{P}}$ in the simulation.

The presence of the canted phase potentially explains two of the features in Fig. 3. First, the canted mode gives insight into the presence of the non-monotonic $I_{\mathrm{C}}^{\mathrm{AP}-\mathrm{P}}$ for $H=-4.5 \mathrm{kOe}$ and the peak in $\mathrm{d} V / \mathrm{d} I$ before reversal (Fig. 4a). The canted mode appears in the simulation in this field range and suggests 


\section{ARTICLES}

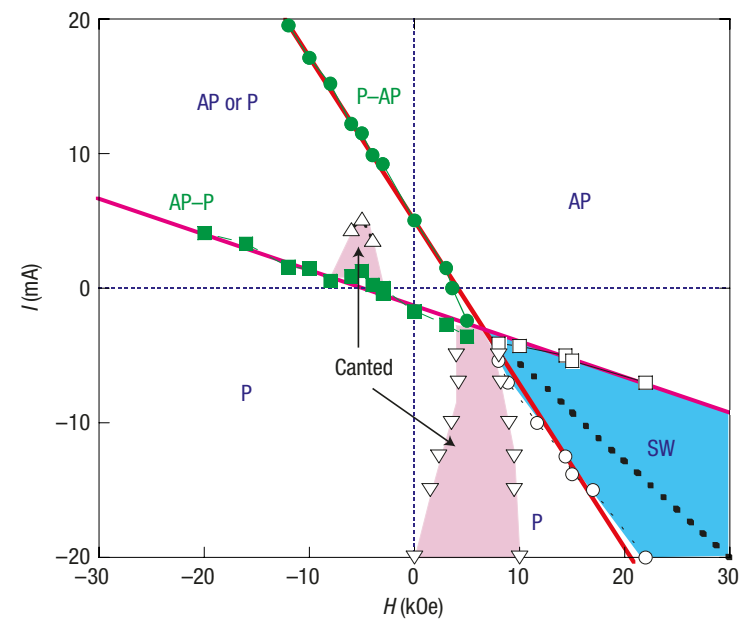

Figure 5 Theoretically derived phase diagram for the switching of the free layer with the magnetization of the reference layer pointing down. The simulation parameters are given in the text. Each symbol corresponds to a distinct change in the resistance with sweeping $I_{\mathrm{B}}$ or $H$. The filled symbols are irreversible changes in the resistance where the circles are $I_{C}^{\mathrm{P}-\mathrm{AP}}$ and squares are $I_{\mathrm{C}}^{\mathrm{AP}-\mathrm{P}}$. The open symbols refer to reversible changes in the resistance where the open squares and circles indicate the onset of uniform spin-wave modes (labelled SW). The bold dashed line indicates the $I_{B}-H$ condition where the average $z$-component of the magnetization switches from parallel to antiparallel in the spin-wave regime. The open triangles bound the region where the magnetization of the free layer is found to cant non-uniformly away from the perpendicular direction. The solid red lines correspond to equation (2).

the reversible canting of the spins facilitates reversal leading to an enhancement in $I_{\mathrm{C}}^{\mathrm{AP}-\mathrm{P}}$. The simulation also finds a reversible reduction of the resistance before reversal that would result in a peak in $\mathrm{d} V / \mathrm{d} I$. Similarly, the presence of this canted mode is consistent with the nearly vertical phase line for $H \sim 4.2 \mathrm{kOe}$ and large negative $I_{\mathrm{B}}$. The boundary of the canted phase $(\nabla)$ in Fig. 5 agrees quantitatively with the vertical phase boundary in Fig. 3 also shown by $(\nabla)$. This reversible transition, which is not related to a processional mode in the simulation, may occur before the onset of precessional modes. These results suggest that the observations of peaks in $\mathrm{d} V / \mathrm{d} I$ may not arise solely from high-frequency magnetic excitation. However, to distinguish the presence of static, nonuniform modes from spin-wave modes experimentally will require time-dependent measurements.

This work provides a demonstration of spin-torque-induced magnetic reversal of perpendicular anisotropy films with high anisotropy. These results are facilitated by the use of a $\mathrm{Co} / \mathrm{Ni}$ multilayer as the magnetic layers that provided high coercive fields and significantly higher GMR ratios and spin-torque efficiencies than we achieved with $\mathrm{Co} / \mathrm{Pt}$ multilayers. The critical currents for reversal of the magnetization are comparable to many in-plane magnetized devices, which typically have lower coercive fields ${ }^{25}$. Compared with the most efficient in-plane GMR devices ${ }^{16}$, the ratio of critical current to the room-temperature coercive field indicate a somewhat improved efficiency for the perpendicular device. However, a quantitative comparison will require determining the appropriate energy barrier to reversal and separating the role of thermal activation. By designing the reference layer with larger anisotropy fields than the free layer, a complete phase diagram could be explored and compared to micromagnetic models. This perpendicular materials system has a remarkable range of magnetic phenomena and may prove suitable for high-density spintronic applications.

\section{METHODS}

The multilayers samples were deposited onto ambient temperature SiN-coated Si wafers combining both sputtering and evaporation techniques in a high-vacuum $\left(10^{-8}\right.$ torr base pressure) deposition system. The reference and free magnetic layers of the $\mathrm{Co} / \mathrm{Ni}$ samples are

$\mathrm{Pt}(3 \mathrm{~nm}) /[\mathrm{Co}(0.25 \mathrm{~nm}) / \mathrm{Pt}(0.52 \mathrm{~nm})] \times$

$4 / \mathrm{Co}(0.25 \mathrm{~nm}) /[\mathrm{Co}(0.1 \mathrm{~nm}) / \mathrm{Ni}(0.6 \mathrm{~nm})] \times 2 / \mathrm{Co}(0.1 \mathrm{~nm})$ and $[\mathrm{Co}(0.1 \mathrm{~nm}) / \mathrm{Ni}(0.6 \mathrm{~nm})] \times 4 / \mathrm{Co}(0.1 \mathrm{~nm}) / \mathrm{Pt}(3 \mathrm{~nm})$, respectively. The reference and free magnetic layers of the $\mathrm{Co} / \mathrm{Pt}$ samples are $\mathrm{Pt}(3 \mathrm{~nm}) /[\mathrm{Co}(0.25 \mathrm{~nm}) / \mathrm{Pt}(0.52 \mathrm{~nm})] \times 4 / \mathrm{Co}(0.25 \mathrm{~nm})$ and $[\mathrm{Co}(0.25 \mathrm{~nm}) / \operatorname{Pt}(0.52 \mathrm{~nm})] \times 2 / \mathrm{Co}(0.25 \mathrm{~nm}) / \operatorname{Pt}(3 \mathrm{~nm})$, respectively. In both cases, the magnetic multilayer structures were grown by electron-beam evaporation. The $4 \mathrm{~nm} \mathrm{Cu}$ layer between the magnetic layers was sputter deposited and the thickness was chosen to avoid coupling between the two magnetic layers. The initial $\mathrm{Ta}(5 \mathrm{~nm}) / \mathrm{Cu}(35 \mathrm{~nm})$ film that acts as the bottom lead and the top $\mathrm{Cu}(15 \mathrm{~nm}) / \mathrm{Ta}(5 \mathrm{~nm})$ film that acts as the top lead and protect the films from oxidation during processing were grown by magnetron sputtering. The resulting films are highly (111) textured.

For device fabrication we used a 100 -nm-thick high-resolution electron-beam resist, HSQ. Electron-beam exposure essentially converts HSQ into $\mathrm{SiO}_{2}$, which provides excellent resistance to ion milling and allows the resist to serve directly as a high-fidelity mask during the etching of the devices. Devices were fabricated both as circles and hexagons. A transmission electron microscopy cross-section image of the finished $\mathrm{Co} / \mathrm{Ni}$ device shows the well-defined layered structure and sample geometry. For the $\mathrm{Co} / \mathrm{Ni}$ devices, $R_{\text {d.c. }}$ of 850 devices was measured. We found that $R_{\text {d.c. }}$ scaled linearly with the inverse of the nominal device area from $1,600 \mathrm{~nm}$ diameter circles down to $50 \times 100 \mathrm{~nm}^{2}$ hexagon samples. For the $50 \times 100 \mathrm{~nm}^{2}$ sample, measuring $R_{\mathrm{d} . c}$. of 47 devices we found a mean of $11.6 \Omega$ with a standard deviation of $0.7 \Omega$. Measurements of $47,100 \times 200 \mathrm{~nm}^{2}$ hexagons found a mean $R_{\text {d.c. }}$ of $2.86 \Omega$ with a standard deviation of $0.3 \Omega$. Similar results were found for the $\mathrm{Co} / \mathrm{Pt}$ devices. A subset of devices was selected for magneto-transport measurements. The current perpendicular to the plane GMR for the $50 \times 100 \mathrm{~nm}^{2} \mathrm{Co} / \mathrm{Ni}$ devices ranged from 0.9 to $1.1 \%$ whereas GMR varied from 0.84 to $1.0 \%$ for the eight devices tested for various sizes. The $\mathrm{Co} / \mathrm{Pt}$ device GMR values ranged from 0.4 to $0.5 \%$.

For transport studies, we measured $R_{\text {d.c. }}$ by measuring the voltage $V_{\text {d.c. }}$ as a function of $I_{\mathrm{B}}$. We also measure the differential resistance $\mathrm{d} V / \mathrm{d} I$ using an a.c. $(\sim 10 \mu \mathrm{A})$ Wheatstone bridge and the d.c. current has been injected using a bias- $T$. In the present geometry, $I_{\mathrm{B}}<0$ means electrons flow from the reference to the free layer. The field and current sweep measurements are quasi-static with typical sweep rates of $10-20 \mathrm{Oe} \mathrm{s}^{-1}$ and $50 \mu \mathrm{A} \mathrm{s}^{-1}$, respectively. Regarding the use of $\mathrm{d} V / \mathrm{d} I$ measurements, most generally $\mathrm{d} V / \mathrm{d} I=R_{\text {d.c. }}+I \mathrm{~d} R_{\mathrm{d} . c .} / \mathrm{d} I$. Assuming that the system is ohmic such that $\mathrm{d} R_{\text {d.c. }} / \mathrm{d} I=0$, then $\mathrm{d} V / \mathrm{d} I=R_{\mathrm{d} . c .}$. If, however, there are current-induced changes in the resistance that are reversible with the a.c. current, we need to include the second term, $I \mathrm{~d} R_{\text {d.c. }} / \mathrm{d} I$, in $\mathrm{d} V / \mathrm{d} I$. This second term can add or subtract to $R_{\text {d.c. }}$ depending on the sign of the slope of $\mathrm{d} R_{\mathrm{d} . \mathrm{c} .} / \mathrm{d} I$ and the sign of $I$. In Fig. 4 a, there is an increase in $\mathrm{d} V / \mathrm{d} I$ before $I_{\mathrm{C}}^{\mathrm{AP}-\mathrm{P}}$ with decreasing $I$ even though $R_{\text {d.c. }}$ is decreasing before $I_{\mathrm{C}}^{\mathrm{AP}-\mathrm{P}}$. This is because $\mathrm{d} R_{\text {d.c. }} / \mathrm{d} I$ is positive and is increasing as we approach $I_{\mathrm{C}}^{\mathrm{AP}-\mathrm{P}}$. Similarly, in Fig. $4 \mathrm{~b}$ we observe sharp dips in $\mathrm{d} V / \mathrm{d} I$. In the data shown in Fig. $4 \mathrm{c}$ for regions where the transitions occur, the slope of $R_{\text {d.c. }}$ versus $I\left(\mathrm{~d} R_{\text {d.c. }} / \mathrm{d} I\right)$ at the transition regions is positive. However, the sign of $I_{\mathrm{B}}$ is negative giving the resulting dips in $\mathrm{d} V / \mathrm{d} I$ arising from $I \mathrm{~d} R_{\mathrm{d} . c .} / \mathrm{d} I$.

For micromagnetic calculations we assumed a $50 \times 100 \mathrm{~nm}^{2}$ hexagon element where both the reference and free layer were $3 \mathrm{~nm}$ thick, each divided into 14 cells and separated by a $3 \mathrm{~nm}$ non-magnetic spacer layer. The reference layer had a saturation magnetization $M_{\mathrm{S}}=500 \mathrm{e} . \mathrm{m} . \mathrm{u} . \mathrm{cm}^{-3}$ and perpendicular anisotropy constant $K_{\mathrm{U}}=2 \times 10^{7} \mathrm{ergs} \mathrm{cm}^{-3}$. The value of $K_{\mathrm{U}}$ is significantly larger than the present samples, but allows us to explore a larger $I_{\mathrm{B}}-H$ space in the calculation without reversing the lower layer. The free-layer parameters are $M_{\mathrm{S}}=650 \mathrm{e} . \mathrm{m} . \mathrm{u} . \mathrm{cm}^{-3}$ and $K_{\mathrm{U}}=3.3 \times 10^{6} \mathrm{ergs} \mathrm{cm}^{-3}$. For both layers the intra-layer exchange between cells was $2 \mu \mathrm{erg} \mathrm{cm}^{-1}$, and $p=0.35$ and $\alpha=0.1$ were assumed. This value of $\alpha$ is larger than that observed typically for Co films, but large effective $\alpha$ values have been inferred for $\mathrm{Co} / \mathrm{Pt}$ multilayers with perpendicular anisotropy ${ }^{28}$. The calculations were carried out assuming finite temperature $(300 \mathrm{~K})$ and sweeping either $I_{\mathrm{B}}$ or $H$. 
Received 4 November 2005; accepted 17 January 2006; published 19 February 2006

References

1. Berger, L. Emission of spin waves by a magnetic multilayer traversed by a current. Phys. Rev. B 54, 9353-9358 (1996).

2. Slonczewski, J. C. Current-driven excitation of magnetic multilayers. J. Magn. Magn. Mater. 159, L1-L7 (1996).

3. Tsoi, M. et al. Excitation of a magnetic multilayer by an electric current. Phys. Rev. Lett. $\mathbf{8 0}$ 4281-4284 (1998)

Myers, E. B., Ralph, D. C., Katine, J. A., Louie, R. N. \& Buhrman, R. A. Current-induced switching of domains in magnetic multilayer devices. Science 285, 867-870 (1999).

5un, J. Z. Current-driven magnetic switching in manganite trilayer junctions. J. Magn. Magn. Mater. 5. Sun, J. Z. Current-driven

6. Katine, J. A., Albert, F. J., Buhrman, R. A., Myers, E. B. \& Ralph, D. C. Current-driven magnetization reversal and spin-wave excitations in Co/Cu/Co pillars. Phys. Rev. Lett. 84, 3149-3152 (2000). reversal and spin-wave excitations in $\mathrm{Co} / \mathrm{Cu} / \mathrm{Co}$ pillars. Phys. Rev. Lett. 84, 3149-3152 (2000).
Kiselev, S. I. et al. Spin-transfer excitations of permalloy nanopillars for large applied currents. Phys. Kiselev, S. I. et al. Spin-tras
Rev. B 72, 064430 (2005).

Xiao, J., Zangwill, A. \& Stiles, M. D. Macrospin models of spin transfer dynamics. Phys. Rev. B 72, 014446 (2005)

9. Gurney, B. A. et al. in Ultrathin Magnetic Structures IV: Applications of Nanomagnetism (eds Heinrich, B. \& Bland, J. A. C.) 149-174 (Springer, Berlin, 2005)

0. Slonczcewski, J. C. Currents and torques in metallic magnetic multilayers. J. Magn. Magn. Mater. 247, $324-338(2002)$

11. Berger, L. Relation between giant magnetoresistance and critical current for spin precession in magnetic multilayers. Phys. Rev. B 72, 100402 (2005).

12. Barnas, J., Fert, A., Gmitra, M., Weymann, I. \& Dugaev, V. K. From giant magnetoresistance to current-induced switching by spin transfer. Phys. Rev. B 72, 024426 (2005).

3. Schmidt, G. Concepts for spin injection into semiconductors-a review. J. Phys. D 38, R107-R122 (2005).

4. Jiang, Y. et al. Substantial reduction of critical current for magnetization switching in an exchange-biased spin valve. Nature Mater. 3, 361-364 (2004).

15. Urazhdin, S., Birge, N. O., Pratt, W. P. Jr \& Bass, J. Switching current versus magnetoresistance in magnetic multilayer nanopillars. Appl. Phys. Lett. 84, 1516-1518 (2004).

6. Braganca, P. M. et al. Reducing the critical current for short-pulse spin-transfer switching of nanomagnets. Appl. Phys. Lett. 87, 112507 (2005).
17. Kent, A. D., Özyilmaz, B. \& del Barco, E. Spin-transfer-induced precessional magnetization reversal. Appl. Phys. Lett. 84, 3897-3899 (2004).

18. Lee, K. J., Redon, O. \& Dieny, B. Analytical investigation of spin-transfer dynamics using a perpendicular-to-plane polarizer. Appl. Phys. Lett. 86, 022505 (2005).

19. Özyilmaz, B. et al. Current-induced magnetization reversal in high magnetic fields in $\mathrm{Co} / \mathrm{Cu} / \mathrm{Co}$ nanopillars. Phys. Rev. Lett. 91, 067203 (2003).

20. Kiselev, S. I. et al. Current-induced nanomagnet dynamics for magnetic fields perpendicular to the sample plane. Phys. Rev. Lett. 93, 036601 (2004).

21. Rippard, W. H., Pufall, M. R., Kaka, S., Silva, T. J. \& Russek, S. E. Current-driven microwave dynamics in magnetic point contacts as a function of applied field angle. Phys. Rev. B 70, 100406 (2004).

22. Özyilmaz, B., Kent, A. D., Rooks, M. J. \& Sun, J. Z. Bipolar high-field excitations in $\mathrm{Co} / \mathrm{Cu} / \mathrm{Co}$ nanopillars. Phys. Rev. B 71, 140403 (2005).

23. Daalderop, G. H. O., Kelly, P. J. \& den Broeder, F. J. A. Prediction and confirmation of perpendicular magnetic anisotropy in Co/Ni multilayers. Phys. Rev. Lett. 68, 682-685 (1992).

24. Hu, G., Thomson, T., Rettner, C. T., Raoux, S. \& Terris, B. D. Magnetization reversal in Co/Pd nanostructures and films. J. Appl. Phys. 97, $10 J 702$ (2005).

25. Lacour, D., Katine, J. A., Smith, N., Carey, M. J. \& Childress, J. R. Thermal effects on the magnetic-field dependence of spin-transfer-induced magnetization reversal. Appl. Phys. Lett. 85, $4681-4683(2004)$

26. http://lgmicro.home.mindspring.com/

27. Lee, K. J., Deac, A., Redon, O, Nozieres, J. P. \& Dieny, B. Excitations of incoherent spin-waves due to spin-transfer torque. Nature Mater. 3, 877-881 (2004).

28. Back, C. H. \& Siegmann, H. C. Ultrashort magnetic field pulses and the elementary process of magnetization reversal. J. Magn. Magn. Mater. 200, 774-785 (1999).

\section{Acknowledgements}

We would like to thank Y. Lemaho, Y. Henry and D. Lacour for assistance in transport measurement and J. Sun for fruitful discussion.

Correspondence and requests for materials should be addressed to E.E.F.

Competing financial interests

The authors declare that they have no competing financial interests.

Reprints and permission information is available online at http://npg.nature.com/reprintsandpermissions/ 